\title{
A peak reduction scheduling algorithm for storage devices on the low voltage network
}

Article

Accepted Version

Rowe, M., Yunusov, T., Haben, S., Singleton, C., Holderbaum, W. and Potter, B. (2014) A peak reduction scheduling algorithm for storage devices on the low voltage network. IEEE Transactions on Smart Grid, 5 (4). pp. 2115-2124. ISSN 19493053 doi: https://doi.org/10.1109/TSG.2014.2323115 Available at https://centaur.reading.ac.uk/36953/

It is advisable to refer to the publisher's version if you intend to cite from the work. See Guidance on citing.

To link to this article DOI: http://dx.doi.org/10.1109/TSG.2014.2323115

Publisher: IEEE

All outputs in CentAUR are protected by Intellectual Property Rights law, including copyright law. Copyright and IPR is retained by the creators or other copyright holders. Terms and conditions for use of this material are defined in the End User Agreement.

www.reading.ac.uk/centaur 
Central Archive at the University of Reading

Reading's research outputs online 


\title{
A Peak Reduction Scheduling Algorithm For Storage Devices On The Low Voltage Network
}

\author{
Matthew Rowe, Student Member, IEEE, Timur Yunusov, Student Member, IEEE, Stephen Haben, \\ Colin Singleton, Member, IEEE, William Holderbaum, Member, IEEE, and Ben Potter, Member, IEEE
}

\begin{abstract}
Reinforcing the Low Voltage (LV) distribution network will become essential to ensure it remains within its operating constraints as demand on the network increases. The deployment of energy storage in the distribution network provides an alternative to conventional reinforcement. This paper presents a control methodology for energy storage to reduce peak demand in a distribution network based on day-ahead demand forecasts and historical demand data. The control methodology pre-processes the forecast data prior to a planning phase to build in resilience to the inevitable errors between the forecasted and actual demand. The algorithm uses no real time adjustment so has an economical advantage over traditional storage control algorithms. Results show that peak demand on a single phase of a feeder can be reduced even when there are differences between the forecasted and the actual demand. In particular, results are presented that demonstrate when the algorithm is applied to a large number of single phase demand aggregations that it is possible to identify which of these aggregations are the most suitable candidates for the control methodology.
\end{abstract}

Index Terms-Energy Storage, LV Network, Forecast, Load Uncertainty, Smart Grid, DNO.

\section{INTRODUCTION}

$\mathbf{T}$ HE electricity demand in the Low Voltage (LV) network is expected to significantly increase in the coming years due to the electrification of transport and heating [1]. In fact, across the network, peak demand is predicted to double by 2050 [2]. An increase in energy demand will require Distribution Network Operators (DNOs) to reinforce their networks to meet this new higher demand. Conventional reinforcement, either by upgrading existing cables and substations or adding new ones, is effective but expensive. At the same time, smart meters are becoming more ubiquitous, giving visibility of the household and low voltage network level demand. This provides the opportunity for new methods of supporting the network such as forecast-based control of storage devices, the topic of this paper. Research in the literature has explored the potential role of storage on the grid [3]-[5] and current projects where storage is currently playing a successful role in the network [6]. Storage has many potential supporting technical applications [7]. One of these potential roles is the time-shifting of energy demand to reduce daily peaks and therefore provide a potentially more cost-effective (and less disruptive) alternative to conventional reinforcement. This

The authors are with the School of Systems Engineering and the Centre for Mathematics of Human Behaviour, The University of Reading, UK

The work has been carried out with Scottish and Southern Energy Power Distribution and funded by the Low Carbon Network Fund established by Ofgem. technique, commonly known as peak demand reduction is the subject of this research presented here.

Research to date in the field on peak demand reduction discusses two possible ways to control a storage device on the distribution network [8]: the first develops a control plan which operates a storage device using a fixed scheduled pattern that is determined in advance. The second control methodology is a load following operation using real time data. Both these techniques run the risks of shortage or surplus of the energy in a storage device particularly due to the volatile nature of the LV network. The first control technique has the advantage of removing the need to rely on monitoring the network, which is expensive. However, the efficacy of a given energy storage device depends on the way it is controlled; energy storage devices based on set-point control, where the battery will discharge or charge when the voltage or frequency of grid at the connection point hit certain values, is fundamentally limited as it operates without any knowledge of what is expected to happen in the future [7], [8]. If demand can be anticipated through load forecasts then a more intelligent control plan can be implemented. Many control algorithms in the literature which have been developed for peak shaving and demand reduction rely on the fact that demand forecasted profiles are $100 \%$ accurate or that every state of the system can be monitored and measured [9]-[11]. This assumption is reasonable when the storage is to be used to mitigate peaks on large aggregations of demand as forecasting errors are relatively small. However, on the LV network with its smaller aggregations of demand, profiles are volatile and difficult to predict. Other algorithms, which endeavour to remove peaks in demand are smoothing algorithms [12] that aim to mitigate variations in demand and generation and algorithms that use pricing signals to reduce demand and give economic benefits to the customer [13]. Xu et al. claim better operation decisions can be made by considering anticipated behaviour and thus introduces demand forecasts into the algorithms [14]. Due to the inherent volatility at smaller aggregations, forecasts at the LV level are more likely to have large errors. These large errors are detrimental to the performance of such control algorithms but can be improved by using the three step control methodology proposed by Molderink et al. [15], the research helps to deal with demand uncertainty by taking a forecast developing a plan and using real time control to deal with the error between forecasts and actual demand for a single household with many controllable elements in the system. The work presented here in this paper will develop control algorithms for DNO owned storage devices who are interested 
in getting the best possible demand reduction from a storage device to reduce losses on a network and reduce the strain on the LV network [16]. Only several papers in the literature focus on the area of controlling a storage device for demand reduction from a network operators view point [17]-[19] and specifically on the LV network [20]. Dinghuan et al. discuss how control algorithms must differ dependant of the location of the storage device on the network [12]. Research has shown that as the price of storage drops, LV connected storage projects with specific applications become cost effective such as community energy storage projects [21] supporting the LV network [22] and mitigating reverse power flows due to PV, peak shifting and voltage support [23].

In this work forecasts and historical demand data are combined at the household level together with an extended three step control methodology to enable peak demand reduction on the LV network. The charging and discharging of the storage device is planned using day ahead forecasts and historical demand data is used to pre-process the algorithm to cope with uncertainty in the forecasts. Real-time adjustments are not considered in this work. In contrast to other work perfect knowledge of future energy demand is not assumed, the work does not rely on accurate and complete real time measurement data and the storage is operated from the viewpoint of a Distribution Network Operator (DNO). Real-time control relies on a communications infrastructure being deployed and maintained. The time and costs required for the communications infrastructure can be reduced if an off-line, planningbased control approach can be shown to provide sufficient network benefits, and sufficient consistency and robustness. Investigating the potential of off-line control approaches is the purpose of this paper. Furthermore, the approach has been developed in close collaboration with Scottish and Southern Energy Power Distribution, the Distribution Network Operator division of SSE in the UK, as part of The New Thames Valley Vision (NTVV) project which is an Ofgem funded Low Carbon Network Fund (LCNF) project, in order to aid DNOs in coping with the increased demand in a future low carbon UK. The project will be deploying twenty-three LV connected storage devices [24]. The Smart Meter data used to generate the forecasts used in this paper have been supplied by the Commission for Energy Regulation in Ireland and are openly available on-line [25]. The forecasts themselves have been generated, and aggregated, to be representative of one phase of one feeder - a likely location for an energy storage device in the distribution network, the aggregations have been chosen to best fit actual substation demand data in order to generate realistic demand profiles, the substation data has been supplied by Scottish and Southern Energy Power Distribution (SSEPD).

This paper has the following structure: Section II describes the novel architecture, Section III presents an example of the control methodology presented on a single demand aggregation, Section IV will show the result of running the algorithm on 500 demand aggregations and the final section, Section V, will discuss and conclude the research.

\section{Methodology}

This work adopts the three stage methodology presented by Molderink et al. which uses the predicted demand and develops a schedule for the storage device and controllable loads and uses this schedule in the real time control [15], [26]. The architecture of the proposed control scheme in this work is shown in Figure 1. However this paper does not consider real time control and hence develops an unchangeable plan for the storage device for the day based on day-ahead forecasts and historical demand data. Section II. A. describes how the forecasts and error bars are produced. The aggregated forecast data (48 half hourly samples per day) are passed to a pre-processing algorithm that in effect filters the forecast to provide an input for the planning stage that will both reduce peak demand and also will avoid causing system violations due to the difference between the forecast and the actual demand. The purpose of this paper is investigate the full potential of off-line, planning-based control of energy storage without real-time control. As previously discussed, the more than can be achieved with off-line control means less investment in infrastructure for real-time control. Therefore, this paper builds upon the off-line, planning-based, elements of the three-stage methodology found in the literature and so does not include the final step that uses real-time data to update the plan. Due to the volatility of the LV network this makes the problem presented in this paper challenging. There are benefits to a DNO for removing this final step which relies on real time data. For example there are significant economic advantages including negating the cost of the monitoring hardware itself and maintaining this hardware and also removing the need to rely on a communication infrastructure to use this data. There are also significant benefits with regards to the speed of deployment.

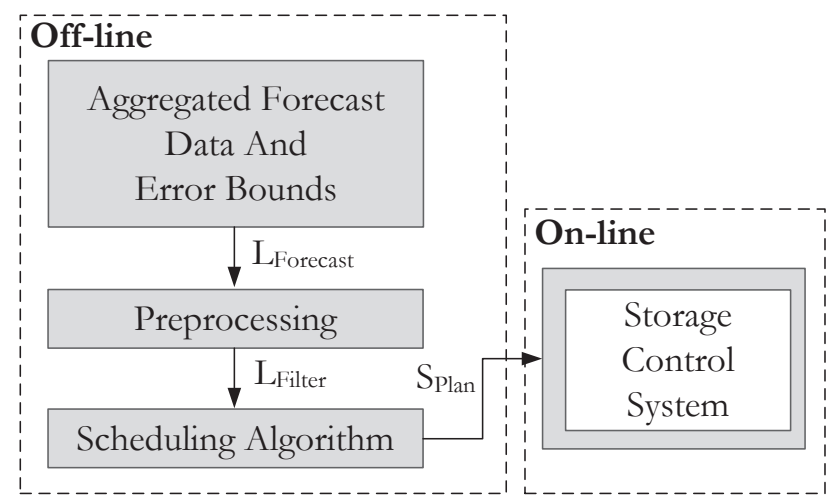

Fig. 1. The architecture of the proposed three step off-line and on line storage control system.

The pre-processing stage, presented in Section II. B, creates a filter $L_{\text {Filter }}$ that operates on the forecast to provide an input for the planning algorithm based on previous statistical data and the forecast; the filter is a function of the forecasts and associated error bounds and is not fixed for each plan. There are many parameters which must be selected to develop the filter 
such as the number of peaks in a forecast and the width and the height of how a forecast peak must vary. These parameters, and how to select them are explained in the following section. The scheduling algorithm, presented in Section II. C, takes the output of the pre-processing filter $L_{F i l t e r}$ and develops a plan for the storage device for the day $S_{\text {Plan }}$. An iterative algorithm has been developed that ensures the storage device is used for the shortest possible time. The planning algorithm contains a model of the battery including constraints such as charge, discharge rate, minimum and maximum storage capacity and the storage device's efficiency. By storing energy in the storage device for the shortest possible time will help to avoid the battery wasted energy through storage leakage and frees up the battery for other storage roles such as voltage support and frequency regulation. This also enables a reduction in the size of the battery and as energy storage is expensive is desirable to get the most out of a given storage units capacity. The storage plan $S_{\text {Plan }}$ is passed to the storage device at the start of a day, shown in the on-line section in Figure 1. The control system then ensures that the device follows the plan by regulating the State Of Charge (SOC) of the storage device. This control system does not use any additional system measurements and deploys the developed plan. If real time data was available $S_{\text {Plan }}$ could be updated on-line.

\section{A. Aggregated Forecasts and Error Bars}

At the household level or LV level energy usage is considerably more volatile than at the Medium Voltage (MV) or High Voltage levels (HV) where large aggregations of customers have smoothed out the daily energy profiles. Hence LV level demand is much more difficult to predict. A large number of forecast methodologies have been developed for application on the MV to HV level demand [27] , [28] but many are not suitable for purposes of this work since they have not been designed to deal with volatile, noisy and irregular LV level usage [29]. Forecasts at the household level have begun to emerge in more recent times as smart meter data becomes more available however such methods remain relatively unsophisticated [30] [31]. Peak usage is likely to differ by a few half hours earlier or later in a normal household due to natural irregularities in behaviour and therefore any forecast to be used for the planning stage of the storage device must allow for these changes [29]. With these points in mind this paper has chosen an innovative simple forecast methodology which assumes week to week regularity but allows for small adjustments in the behaviour. In this paper the forecasting approach previously developed and published by the authors is outlined below, but more details are available in [29]. In the forecast it is assumed that the data is at a half hourly resolution but the method applies to other resolutions. An initial baseline profile $\mathbf{F}^{(1)}$ is produced for the $d^{\text {th }}$ day of the week. Each half hour in this profile is a median of the previous $N$ profiles of the $d^{\text {th }}$ day of the week at the same half hour. Let $\mathbf{F}^{(i)}$ be the baseline profile for the $i^{\text {th }}$ iteration of the algorithm and $\mathbf{G}^{(i)}$ be the profile for the $d^{\text {th }}$ day from $i$ weeks prior to the forecasted. The restricted permutation of the current historical profile, $\hat{\mathbf{G}}^{(i)}$, is then found which minimise the 4norm distance between $\mathbf{F}^{(i)}$ and $\mathbf{G}^{(i)}$. A restricted permutation is one which allows the permutation of each of the points in the historical profile by a fixed number of half hours either side of its original placement. In this case it is allowed a permutation of 3 data points (or half hours) either side. The baseline is updated as an average of the initial baseline and all the current permuted baselines:

$$
\mathbf{F}^{(i+1)}=\frac{1}{i+1} \sum_{j=1}^{i}\left(\mathbf{F}^{(1)}+\hat{\mathbf{G}}^{(j)}\right)
$$

The forecast is then defined to be the final baseline $\mathbf{F}^{(N+1)}$. As well as being inexpensive to calculate, the advantage of this algorithm is the preservation of strong regular peaks, which may change in time by a few half hours from week to week and therefore captures regular but different timed behaviour more effectively than a simple average over the $N$ historical profiles. No location data exists with the household usage data from the Irish smart meter data, hence for the forecasts used in this work it is not possible to consider the effect of weather variables on the energy usage. The average weather data over the whole of Ireland was not considered since the variation across the country may lead to more inaccurate forecasts for some households. For the confidence intervals on the forecasts for each half hour, the lower and upper quartiles of the historical data of that half hour as a lower and upper bound respectively is used. Hence $50 \%$ of the historical data is within these bounds. In future work more sophisticated forecast methodologies and confidence interval estimation will be developed which also take into account external attributes such as temperature and humidity. These individual forecasts form the aggregations discussed in the following sections.

\section{B. Pre-processing}

The preprocessing algorithm aims to increase the certainty that the storage device, when run with no real time data, is still able to substantially reduce the peaks even when inevitable deviations appear between forecasts and actuals. The algorithm seeks to find a filter $\left(L_{\text {Filter }}\right)$ using the forecast and historical demand data, that will widen the peaks and increase their height following a set of rules, this increases the robustness of the plan in coping with uncertainty in the forecasts. Figure 2 shows the 3 stages of the pre-processing algorithm, the forecast is increased in magnitude, width and in the final step smoothed. Figure 3 shows an example of a forecasted peak with the information that is required in the preprocessing stage to develop a filter. There are three stages to the filtering process, as shown in Figure 2 and the algorithms are presented, these stages are represented as $F_{R 1}$, $F_{R 2}$ and $F_{R 3}$, for stages 12 and 3 respectively, the final output $F_{R 3}=L_{\text {Filter }}$. Each consecutive stage uses the previous stages output as that stages input. The algorithm for finding $F_{R 1}, F_{R 2}$ and $F_{R 3}$ can be found in, Section II. B. 1, Section II. B. 2 and Section II. B. 3 respectively. 


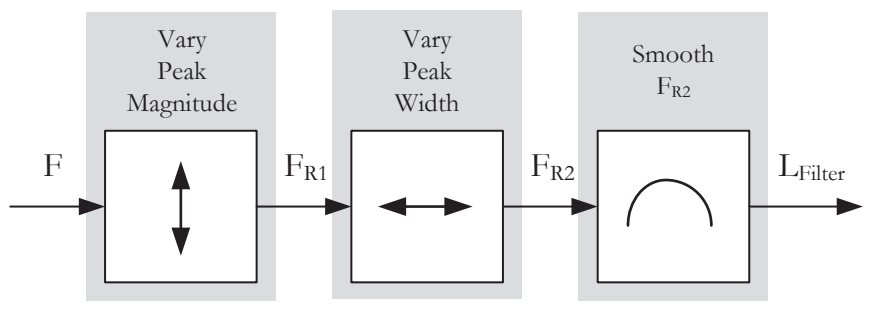

Fig. 2. Diagram showing the 3 stages of the pre-processing algorithm, the forecast $F$ is varied in magnitude, width and smoothed to form $L_{F i l t e r}$

\begin{tabular}{|l|l|}
\hline Variables & Parameters \\
\hline$F=$ Forecast Vector & $W_{\text {Peak }}^{+}=$Width of new peaks (Late) \\
$M_{P}=$ Mid Point Vector & $W_{P e a k}^{-}=$Width of new peaks (Early) \\
$U_{b}=$ Upper Bound Vector & $N_{P e a k}=$ Number of Peaks \\
$L_{b}=$ Lower Bound Vector & $A_{P e a k}=$ Amount to increase peak by \\
$F(k)=$ Forecast at time k & $N_{O}=$ Order of the filter in $F_{R 3}$ \\
\hline
\end{tabular}

TABLE I

TABLE SHOWING PARAMETERS AND VARIABLES USED IN FILTERING ALGORITHM

The input to the Pre-processing algorithm (shown in Figure 3 ) is the forecast $F$ and the output (after stage 3) $F_{R 3}$ is set equal to $L_{\text {Filter }}$. The pre-processing stage creates a filter $L_{F i l t e r}$ that adapts the forecast to provide an input for the planning algorithm based on previous statistical data and the forecast. Figure 3 shows an example of the peak being widened and increased in magnitude, after stage $2\left(F_{R 2}\right)$. The filter peak can be increased in height by $A_{P e a k}$ and width by $W_{P e a k}^{+}$and $W_{P e a k}^{-}$. The parameters in Table 1 are found using a parameter selection process introduced in Section.II.D.

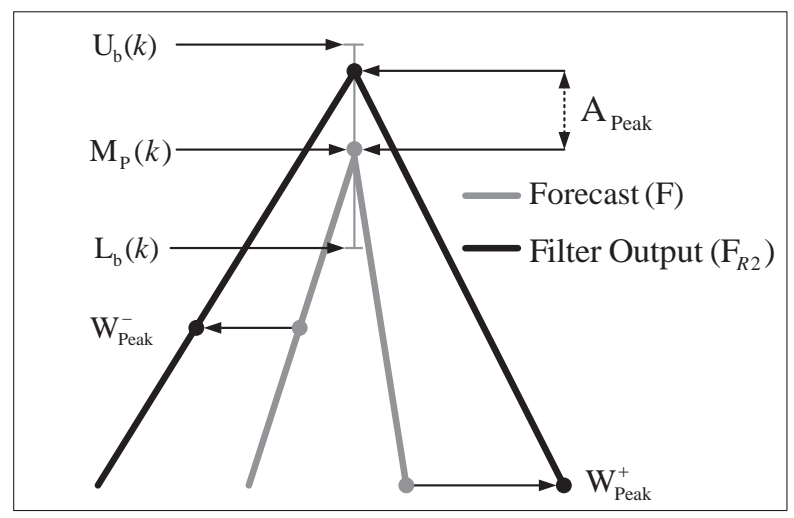

Fig. 3. Diagram shows the filter after stage 2 where the forecast has been changed in magnitude and width, the parameters are shown in table 1

1) Increase Peak Height, Find $F_{R 1}$ : Stage 1 finds a filter $F_{R 1}$ which adjusts the forecasted selected peaks based on where a value lies within the error bounds. $M_{\text {Point }}$ is the midpoint between the boundaries shown in Equation (2). There are two conditions shown in Equation (3), $N$ is the number of samples used to form the forecast where $k=1, \ldots, N$. The forecast height is increased to mitigate the potential error in magnitude that may occur between the forecast and the actual. Setting the incorrect value for $A_{P e a k}$ will waste storage capacity or risk not reducing the peak to the greatest potential of the storage device.

$$
\begin{gathered}
M_{P}(k)=\frac{U_{b}(k)-L_{b}(k)}{2} \\
F_{R 1}(k)=\left\{\begin{array}{c}
{\left[\left(1-\frac{U_{b}(k)-F(k)}{U_{b}(k)-M_{P}(k)}\right) A_{P e a k}\right] F(k),} \\
\text { if } F(k)-M_{P}(k)>M_{P}(k) \\
{\left[\left(\begin{array}{c}
\left(1-\frac{M_{P}(k)-F(k)}{M_{P}(k)-L_{b}(k)}\right) A_{P e a k} \\
\text { if } F(k)-M_{P}(k) \leq M_{P}(k)
\end{array}\right] F(k),\right.}
\end{array}\right.
\end{gathered}
$$

The farther the forecast is from the upper or lower bound the more effect $A_{P e a k}$ has on the filter. If $F(k)$ is far from a bound then $A_{P e a k}$ will have a large effect as it is likely this forecast is not representative of historical data and the storage device needs to be aware the peak is less likely to appear as forecasted.

2) Increase Peak Width, Find $F_{R 2}$ : Stage 2 finds a filter $F_{R 2}$ using $F_{R 1}$ that increases the width of peaks so that if the forecasted value is early or late the storage is still able to minimise the peak. As previously discussed the forecasting methodology used a restricted permutation which allows the permutation of each of the points in the historical profile by a fixed number of half hours either side of its original placement. Ensuring the width of the peaks are increased less than that of the window used in the restricted permutation in the forecasting methodology, will decrease the likelihood of the storage device charging on an early peak. The filter widens the peak by $W_{P e a k}^{+}$and $W_{P e a k}^{-}$as shown in Figure 2. The algorithm is shown below and iterates $N_{P e a k}$ times until the filter $F_{R 2}$ contains all widened peaks. Section II.D, the parameter selection algorithm, explains how $N_{k}, W_{P e a k}^{+}$and $W_{P e a k}^{-}$are found, the pseudo code for finding $F_{R 2}$ is presented in algorithm 1 . The function on line 5 , WidenPeakLate, takes the peak in $F_{R 1}\left(\mathcal{P}_{\text {Max }}\right)$ and widens the peak by $W_{\text {Peak }}^{+}$. The final function RemovePeak removes peak $\mathcal{P}_{\text {Max }}$ from the previous filter $F_{R 1}$ so the next iterations of the algorithm acts on the next peak.

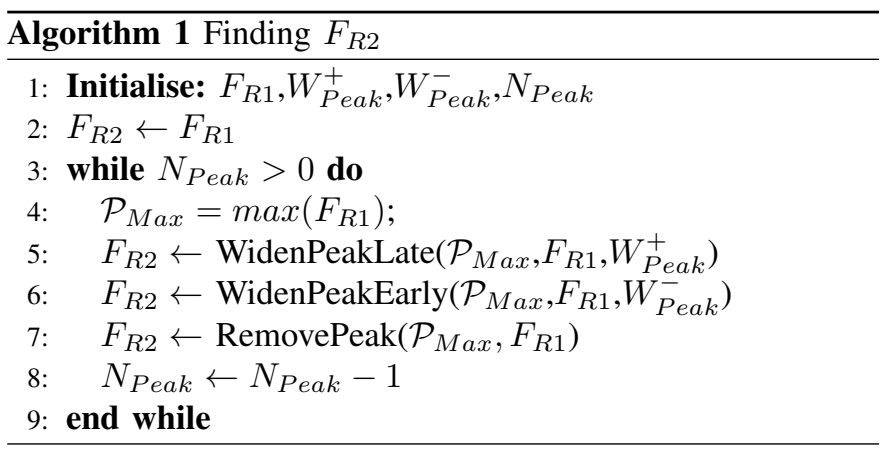

3) Smooth $F_{R 2}$, Find $F_{R 3}$ : The final stage convolves filter $G\left(N_{O}\right)$, Equation (4), with $F_{R 2}$, therefore $F_{R 3}=G\left(N_{O}\right)$ * $F_{R 2} . Z(k)$ in filter $G\left(N_{O}\right)$ contains the coefficients of the filter, where $N_{O}$ is the order of the filter [32]. The algorithm uses a polynomial least squares regression of order initial size 


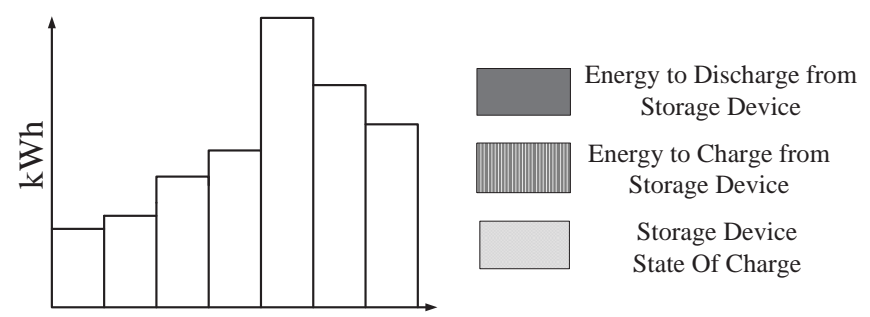

- Filter L Filter $_{\text {}}$

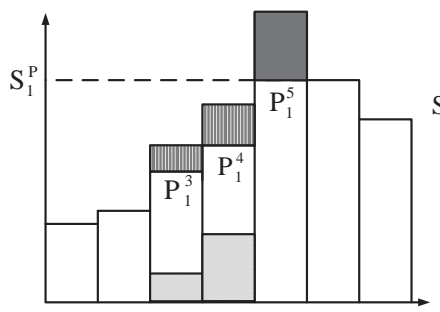

Iteration $1 \mathrm{~F}_{1}$

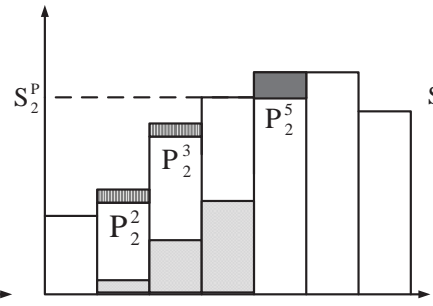

Iteration $2 \mathrm{~F}_{2}$

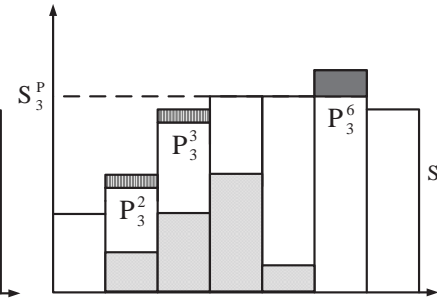

Iteration $3 \mathrm{~F}_{3}$

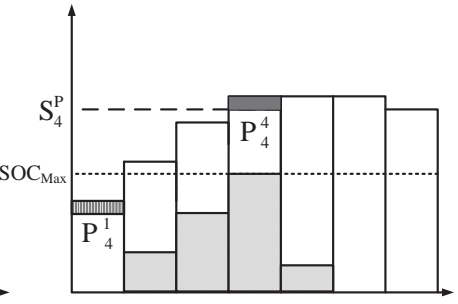

Iteration $4 \mathrm{~F}_{4}$

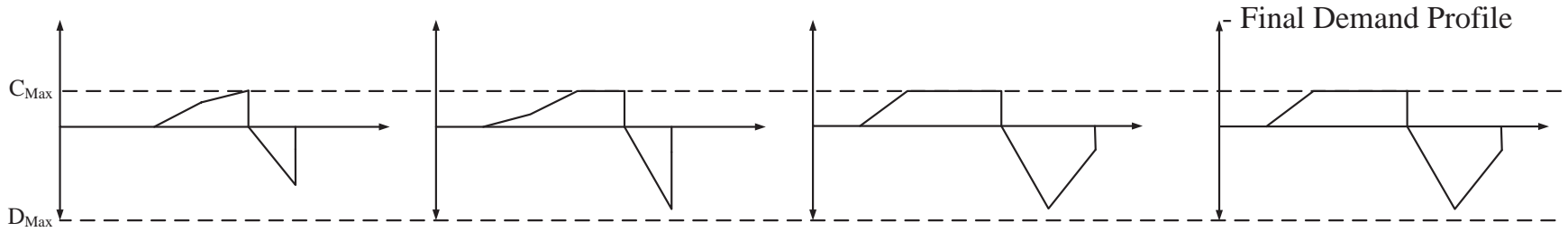

Fig. 4. Diagram graphically showing 4 iterations of the scheduling algorithm, at each iteration the required Set-point is shown and the energy required to charge and discharge to get the profile to reach this set-point is presented. The bottom plots show the required charge and discharge rates of a charge device to achieve the desired reduction

$n$ to find the filter coefficients $Z(k)$ where $x=\left\{1, \ldots, N_{O}\right\}$, finally $L_{\text {Filter }}=F_{R 3}$.

$$
G\left(N_{O}\right)=\sum_{k=1}^{N_{O}+1} Z(k) x^{k}
$$

\section{Scheduling algorithm}

The scheduling algorithm takes the output of the filter from the preprocessing algorithm, discussed in the previous section, of $k$ samples and finds the optimal storage plan for the day that reduces the peak demand to the smallest value using a given finite sized storage device. The following parameters are used throughout the algorithm.

i Iteration number

$\epsilon \quad$ Error Tolerance

$M \quad$ Maximum Number of iterations

$N \quad$ Number of Samples

$S_{i}^{P} \quad$ Set-Point during iteration $i$

$P_{i}^{k} \quad$ Peak Number during iteration $i$ at sample $k$

$C_{\max } \quad$ Maximum Charge Rate

$D_{\max } \quad$ Maximum Discharge Rate

$S O C_{\max }$ Maximum State Of Charge (SOC)

$E_{\max } \quad$ Max Vector, where $i^{t h}$ component

is max value at $i^{\text {th }}$ iteration

The algorithms objective is to find the smallest maximum demand for a given aggregation, using a specific predefined storage device given:

$$
\begin{gathered}
K=\{1, \ldots, N\} \\
F_{i}=\left(a_{i}(1), \ldots, a_{i}(N)\right) \in \mathbb{R}^{1 \times N}, \forall i \in\{1, \ldots, M\}
\end{gathered}
$$

Where $F_{i}$ is the energy profile at iteration $i$, of length $N$ samples. The profile has evenly spaced intervals of $\delta k$, where $a_{i}(k)$ energy is used in sample $k$ of iteration $i$ of the Scheduling Algorithm Iteration (SAI). The complete algorithm is presented below, $F_{1}$ is initialised to $L_{\text {Filter }}$.

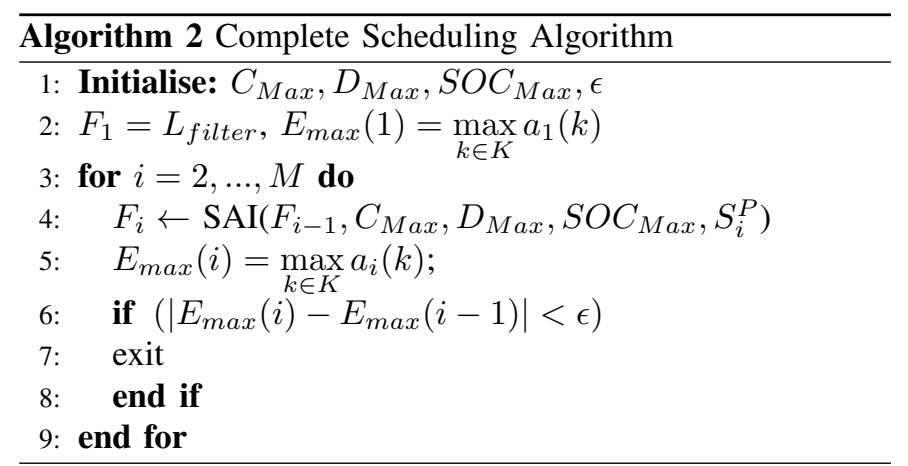

The algorithm will run for $\mathrm{M}$ iterations or until the difference between the current iteration and previous iteration is less than the error tolerance $\epsilon$. The function SAI is one iteration of the scheduling algorithm explained below and is subject to constraints on the storage device: maximum charge rate $C_{M a x}$, maximum discharge rate $D_{M a x}$ and maximum SOC $S O C_{\text {Max }}$. At each iteration $i$ the algorithm attempts to minimise the peak to the set-point $S_{i}^{P}$ which, is set at the size 
of the second largest peak in the demand profile during the current iteration. The optimal storage plan will store energy in a storage device for the shortest possible time. In order that the available storage space is then available for other storage roles to support the network, such as voltage support or frequency regulation, keeping the size of the battery down and not wasting the limited storage capacity available. By storing energy in the storage device for the shortest possible time, it will help to avoid the battery wasting energy through storage leakage. This also enables a reduction in the size of the battery and as energy storage is expensive is desirable to get the most out of a given storage units capacity. It is also expected traditional peak and off peak times are likely to be less common as low carbon devices, such as electric vehicles with overnight charging and heat pumps change the demand behaviour on this part of the volatile LV network, meaning the algorithm will be resilient to expected changes in future demand behaviour, by not relying on a specific temporal demand restriction. The algorithm achieves being able to store energy for the shortest possible time by back filling the demand profile and only storing energy in the storage device during consecutive samples before the peak. An example is shown in Figure 4. The function SAI in algorithm 2 line 4 will run one iteration $(i)$ of the scheduling algorithm and output the resultant energy profile after the iteration. In Figure 4 at iteration 1 the algorithm attempts to distribute the energy above $S_{1}^{P}$ at $P_{1}^{5}$ across $P_{1}^{4}$. At the previous peak $\left(P_{1}^{4}\right)$, the maximum charge rate restricts the amount of energy that can be supplied by the storage device so the remaining energy is charged at $P_{1}^{3}$. The resultant power flow in and out of the storage device to reduce this peak to the set-point and the storage SOC is shown. The example takes 4 iterations to converge as to reduce the demand to $S_{4}^{P}$ in iteration 4 would require violating the $S O C_{\text {Max }}$ constraint to reduce the profile to the set-point. The final result shows the demand has been reduced to $S_{3}^{P}$. When the demand cannot be reduced to the setpoint but the storage device constraints have not been violated, the algorithm iteratively reduces the set-point of that iteration until it can be achieved. The algorithm assumes the storage device is $100 \%$ efficient. The storage device profile in the final result is $S_{P l a n}$ and is deployed to the storage device and will remain unchanged.

\section{Parameter Selection}

During the results section it will be discussed that selecting parameters based on an aggregations historical demand data has shown to successfully generate a set of parameters to use to control the storage device. The filter is a function of parameters $W_{P e a k}^{+}, W_{\text {Peak }}^{-}, N_{\text {Peak }}, A_{\text {Peak }}$ and $N_{O}$. It is assumed that $W_{P e a k}^{+}=W_{P e a k}^{-}$so the peaks can only be widen the same amount on each side and the order of the polynomial filter $\left(N_{O}\right)$ is set to six. This has been shown to be the smallest polynomial degree to give the best fit given the volatile demand data found on a single phase of a Low Voltage network. On multiple runs of the algorithm and varying the parameters in a predefined set it is possible to find the set of parameters which achieves the best demand reduction on average across all historical data. This is carried out independently of the final set of data used to test the parameters selected in the off line architecture. Algorithm 3 shows the parameter selection process. The following variables need to be initialised $W_{\text {Peak }}^{\text {Max }}$ is the maximum amount the width of the peak is to be increased by, $N_{P e a k}^{M a x}$ is the maximum number of peaks to be used, $A_{\text {Peak }}^{M a x}$ is the the maximum magnitude the peak can be increased by, $N_{O}^{M a x}$ is the order of the polynomial applied in step 3 of the pre-processing algorithm and $D^{\text {Max }}$ is the maximum number of days of historic data to be used to generate the parameter set respectively. Placing an upper limit on the parameters means the algorithm can iterate through all possible parameter combinations. Therefore, to maximise the search space, the parameters can be initialised to their maximum values. $F_{d}$ contains the demand profile for day $d$. The GetParameter function on line 16 then returns the set of parameters which occurs most frequently to gain the greatest demand reduction throughout the historic data. These parameters are then used to regenerate $L_{\text {Filter }}$ to find the storage plan to be deployed the following day.

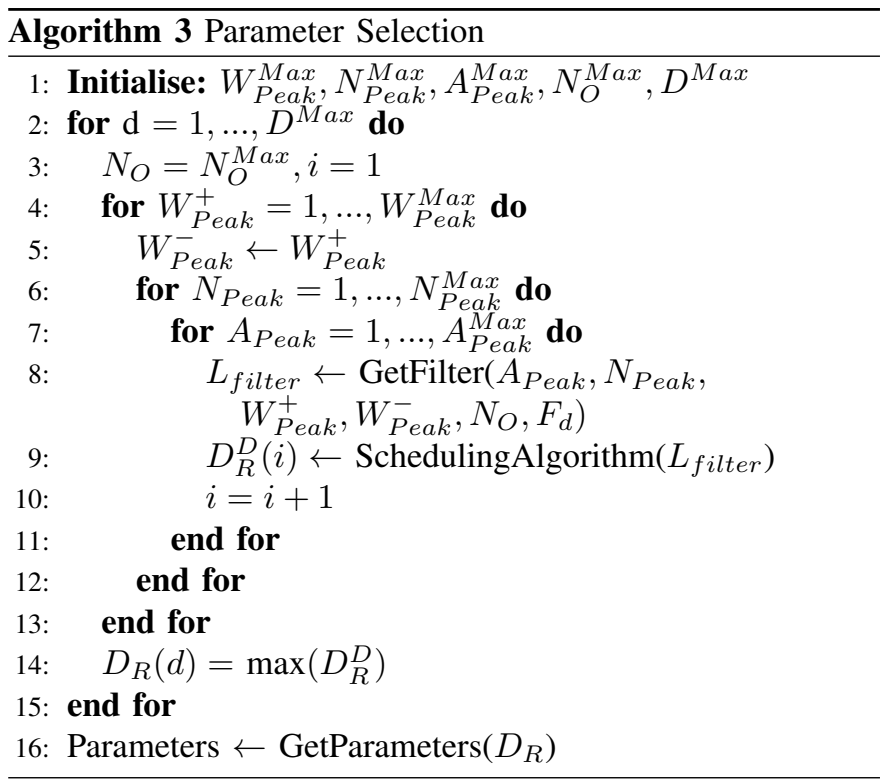

\section{RESULTS AND DISCUSSION FOR THE SCHEDULING} ALGORITHM AND AN INDIVIDUAL AGGREGATION

This section will present a result from the scheduling algorithm, the complete off-line architecture on an individual demand aggregation and then run on a larger dataset of 500 single phase demand aggregations.

An example of the fully implemented scheduling algorithm is presented and the associated results are shown below. Figure 5 shows results from a storage device with a maximum discharge rate of $3 \mathrm{~kW}$ and charge rate of $2.5 \mathrm{~kW}$ over a sample $(k)$ with a maximum storage size of $3 \mathrm{kWh} . \epsilon=0.001, M=500$, $N=48$ and $\delta k=30$ minutes. The peak demand has been reduced from $6.5 \mathrm{kWh}$ to $3.5 \mathrm{kWh}$ (a $46 \%$ reduction) and the storage device capacity is used for the shortest possible time without violating the storage device constraints this algorithm ran for 70 iterations $(i)$. 

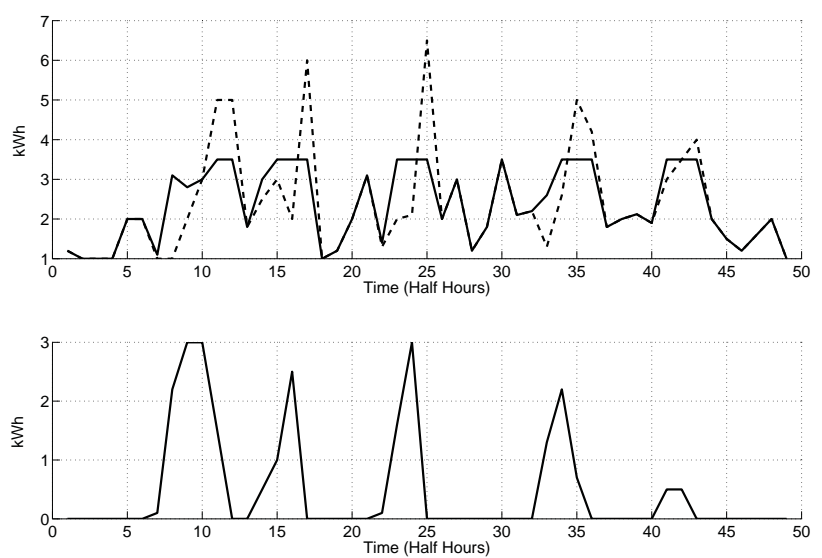

Fig. 5. Result 1 of the iterative algorithm - Top plot: Dotted line is the forecast, Solid line is the forecast after the algorithm is run. Bottom plot: The storage plan SOC profile $S_{\text {Plan }}$

Thirty homes, represented by smart meter data, were aggregated to represent a realistic single phase of a feeder in the distribution network and validated against historical substation data. A period of 19 weeks was considered and split into 14 weeks of historical data and five weeks of future data; the forecasts were based on the first 14 weeks. An example of a forecast for a given day in the 5 week period is shown in Figure 6 . The shaded area shows where $50 \%$ of the historical data fits and therefore represents the upper and lower bounds supplied with the forecast, as previously discussed.

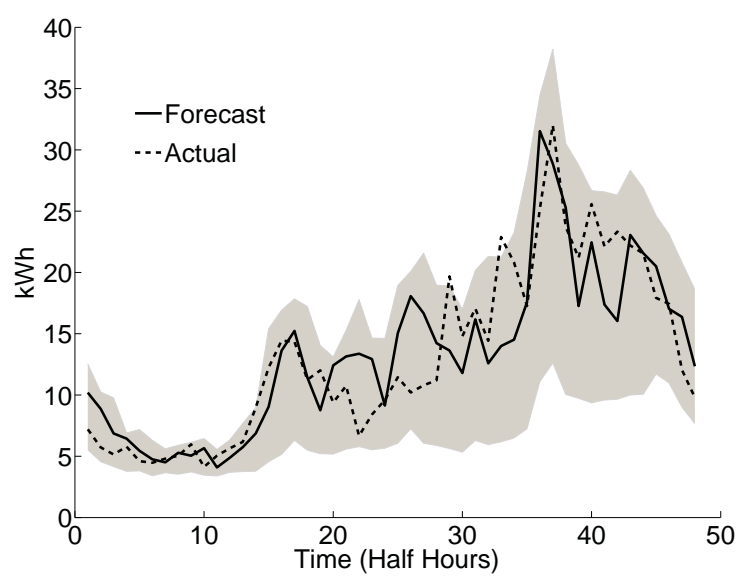

Fig. 6. Plot shows the forecast for one day of 30 aggregated loads. The error bounds have been calculated using 14 weeks of historical data. The shaded area shows where $50 \%$ of the historical data fits

Figure 7 shows the results of running the proposed control scheme on the same day. The following parameters are used in the scheduling algorithm, $\epsilon=0.0001, M=1000, N=48$ and $\delta k=30$ minutes. A storage device with a rated capacity of $25 \mathrm{kWh}$ has been used with a maximum charge rate of $15 \mathrm{~kW}$. The figure also shows both the original demand profile for the day and the plan produced by $L_{\text {Filter. }}$ The peak demand has been reduced by $19 \%$ using the plan generated using filter

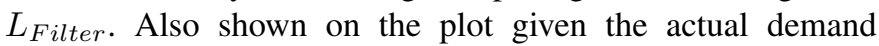
profile for that day. The bottom plot shows the SOC of the
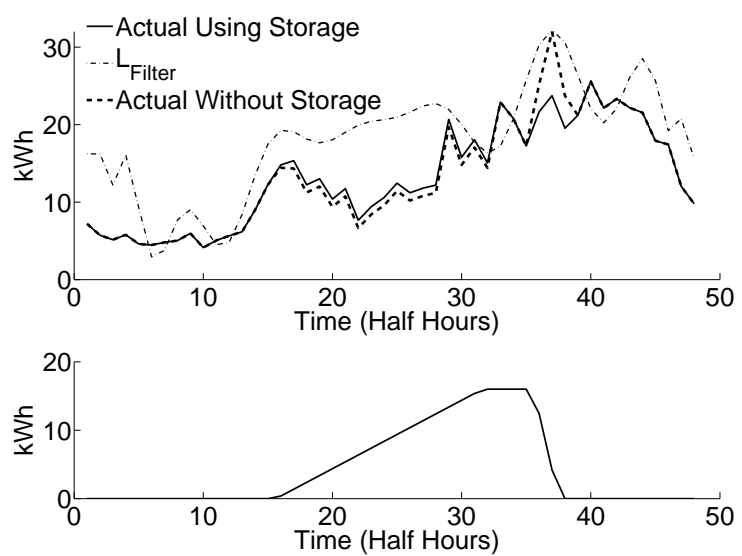

Fig. 7. Top: Simulation Results showing the day with and without storage. Bottom: SOC of the Storage device during the On-line period $\left(S_{P l a n}\right)$

storage device, as determined by the storage plan generated from $L_{\text {Filter. }}$. The device charges between half hour 15 and 35 and discharges between half hour 36 and 38, this corresponds to the storage device discharging to reduce the peak in the top plot at the same time. As a point of comparison, if the forecast error had been zero for the same day, the maximum possible reduction of peak demand would have been $24 \%$, found using the scheduling algorithm on the actual demand data. By running this simulation over the entire 5 weeks of future data a percentage of certainty is generated to demonstrate the effectiveness of the approach over a range of different types of daily energy demand: a reduction in peak demand is achieved $97 \%$ of the time. Furthermore, there is a 55\% chance the peak reduction will be at least $10 \%$. The following parameters are used in the day shown in Figure 7, $A_{\text {Peak }}=0.5 \mathrm{kWh}$, $N_{O}=6, W_{P e a k}^{+}=W_{P e a k}^{-}=1$ and $N_{\text {Peak }}=3$. These parameters are found by running the algorithm over all sets of the parameters ( $N_{O}$ is kept constant) for each historical day in the data set, as described in the parameter selection process in Section II.D, the set of parameters that gives the greatest demand reduction on average across the historical data are used for the following day and the plan generated is shown in Figure 7.

\section{RESUltS AND DiscusSiOn FOR LARGER DATA SETS}

The off-line architecture is now run on 500 individual demand aggregations, the example in the previous section was for just one aggregation. The Smart Meter data used to generate the forecasts has been supplied by the Commission for Energy Regulation in Ireland and is openly available online [25]. The following experiments use 20 weeks of historical data. As above for each aggregation the first 14 historical weeks are used for forecasting, weeks 15-18 are presented. The final weeks (weeks 19-20) are used for the final experiment.

So that the demand reduction is comparable between aggregations the storage device is sized at $25 \%$ of the maximum peak of a demand profile $(F)$ in $\mathrm{kWh} \max (F) \times 0.25$. Then using the scheduling algorithm with $D_{\max }=\max (F) \times 0.25$ and $C_{\max }=D_{\max } / 2$ the best possible demand reduction using the actual demand data for all 4 weeks of test data 
(weeks 15-18) is found. Figure 8 shows 7 box plots representing each day of the week. The maximum demand reduction is $25 \%$ given the how the storage device has been sized. The median sits between $16 \%$ and $18 \%$ across the 7 days when 500 single phase LV network aggregations of 15 smart meter demand profiles are studied using the off-line algorithm. This result shows the volatile nature of the LV network as multiple peaks restrict the storage device from reducing the demand by $25 \%$ in certain aggregations. As an example the Inter Quartile Range (IQR) is between $14 \%$ and $23 \%$ showing where $50 \%$ of the demand profiles maximum reductions fit in the complete dataset.

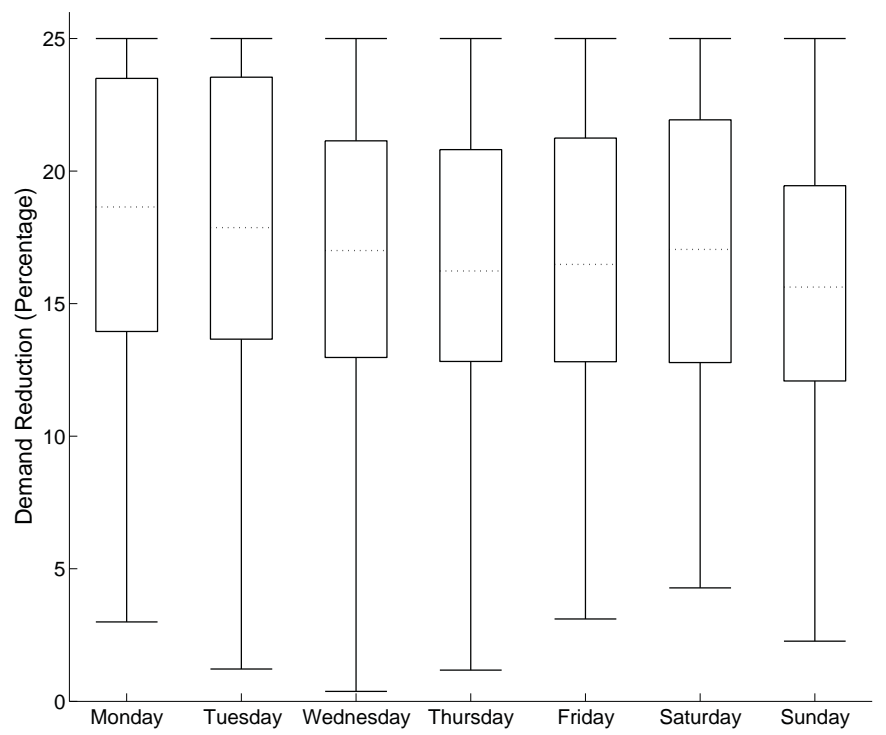

Fig. 8. Box plots per day of the best possible demand reduction

The algorithm is now run on the 4 test weeks (weeks 15 18) of data using the same 500 aggregations, and all the previous historical weeks with forecasts are used for parameter selection. A Probability Density Function (PDF) is presented showing the distribution of the peak demand for each day of the week for each aggregation. The result is shown in Figure 9, each distribution represents a different day of the week using all test data. The result shows a distribution where the mean is approximately zero across all 500 demand aggregations.

There are a number of factors that contribute to this distribution of results, these are due to the volatility of the LV network as previously discussed, and forecasting errors which occur due to smaller aggregations of demand being more difficult to predict. A negative demand reduction can be caused due these errors and the difficult nature of this part of the network to predict. The negative results occur when the storage device charges and causes a new maximum peak. As a comparison, the scheduling algorithm has been compared to a planningbased version of set-point control, derived from [8], [33]. As per [33], the set-point value is determined from historical data, as is typical of other set-point approaches in the literature. To enable a comparison of algorithms, and not off-line vs. real-time storage control, the set-point control algorithm is applied to the same forecast $(F)$ that is fed to $F_{R 1}$ in the

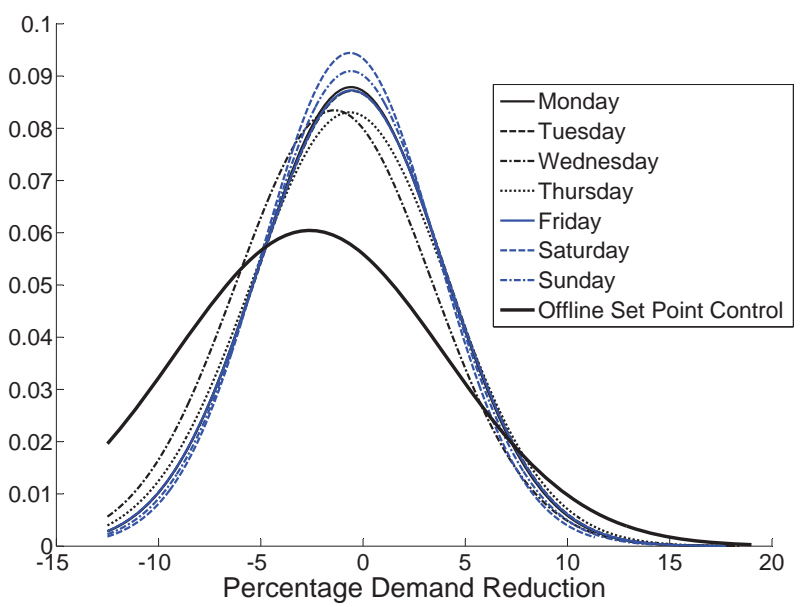

Fig. 9. A Probability Density Function showing the demand reduction as a percentage over a 4 week period using the off-line algorithm presented in this paper run on 500 single phase demand aggregations

pre-processing algorithm. A plan using the set-point based algorithm for the day-ahead is generated and as with the algorithm presented in this paper the storage plan developed is deployed to the storage device and remains unchanged throughout the day. Figure 9 shows the distribution of the performance of the planning based set-point control algorithm when run on the same demand profiles found in the other seven distributions. The off-line set-point control performance distribution shows the mean is less than that of the scheduling algorithm and a negative demand reduction is more likely to occur shown by the shape on the distribution.

An important step of the work is to find the aggregations that do not perform consistently well and remove these aggregations from the complete data set for comparison. It must be possible to do this only from historical data, an initial attempt at this is proposed as follows. Any aggregation from weeks $15-18$, in the data previously presented, who performed badly are removed (had at least one negative result), all historical data previous to week 19 is then used for parameter selection on the remaining subset of aggregations and weeks 19-20 are presented. Other more formal techniques to find these aggregations will be studied in the future, taking into account historical forecast errors, volatility measures and extraneous conditions such as temperature variations. Analysis has shown there is not a direct relationship between the accuracy of the forecast and how well an aggregation performs using the scheduling algorithm, therefore finding suitable candidates from the complete dataset based on forecast performance alone is not always suitable, this is due to how difficult it is to forecast this part of the network. It is expected that as new daily smart meter data becomes available to the DNO the suitability of the algorithm for a specific demand aggregation will need to be re-evaluated. Figure 10 shows the mean of all data from weeks 15-18 from Figure 9 and a distribution curve for the final 2 weeks of data (weeks 19-20) for each day is shown, it is possible to see that removing the aggregations that have not historical performed well under this control 
methodology yield a positive mean result for each day of the 2 weeks of test data. The demand aggregations medians and Inter Quartile Ranges are all positive, though several negative results can still be found. The aggregations in this subset are key candidates for the off-line control methodology presented in this research. The performance of the selection process when using the off-line set-point control algorithm as previously discussed is evaluated. There are significantly fewer candidates found than compared to using the peak reduction algorithm presented in this paper. This result not only shows a direct benefit of using the control scheme presented in this paper over a comparable control technique but reinforces the fact that the subset of aggregations are not only highly forecastable aggregations. If this was in-fact a subset of highly forecastable aggregations and the aggregations could be selected based on forecast performance alone, the off-line set-point control algorithm would have been expected to perform as well as the algorithm presented in this paper. The off-line set-point control algorithm charges in forecasted areas of low demand and discharges during forecasted areas of high demand, defined by a set-point found from the a priori data, and given an accurate forecast would be expected to have a satisfactory performance. Therefore a DNO could potentially evaluate an aggregations historical demand profile prior to deploying the storage device to find whether the aggregation is an ideal candidate for this control methodology. This comparison has shown that not all 500 aggregations would be suitable candidates but it is possible to find, based on historical data, a set of aggregations that may be ideal candidates for the control methodology.

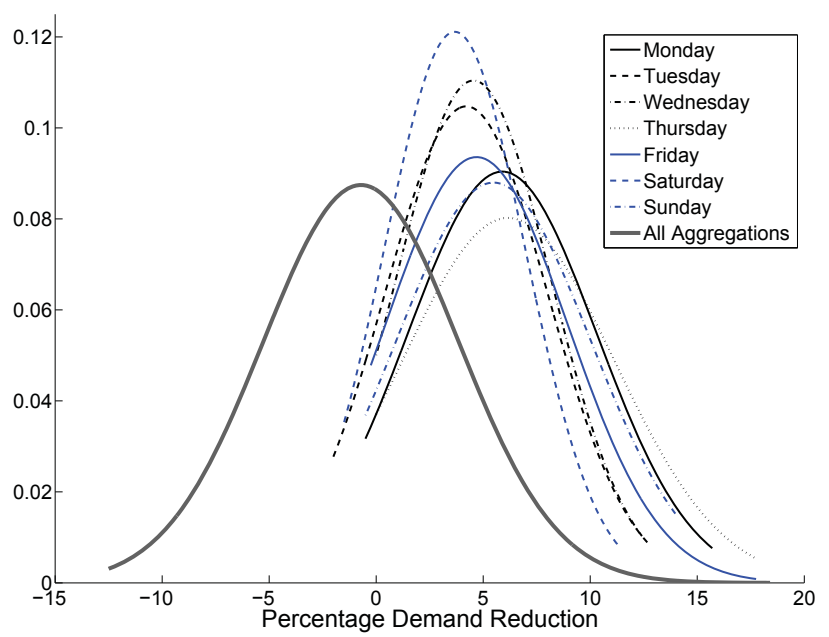

Fig. 10. A Probability Density Function showing the demand reduction as a percentage over a 2 week period using the off-line algorithm presented in this paper showing a subset of the complete 500 demand aggregations, selected from historical demand data

\section{CONCLUSiON}

This research has presented a control scheme for energy storage devices in the distribution network to reduce peak demand based on day-ahead demand forecasts. The control scheme takes a day-ahead forecast complete with error bounds, pre-processes in a filter stage and then develops a plan for the day to reduce peak demand whilst also minimising the time the energy is stored for. The approach does not rely on fully accurate forecasts or real-time system-wide monitoring. Results presented in the paper demonstrate for a specific case study that the peak in the energy demand on a single phase of a feeder can be reduced by over $10 \%$ more than $55 \%$ of the time and a reduction in peak demand is achieved $97 \%$ of the time. Although it is expected that an operational energy storage device will use additional real-time control strategies, the work presented here has demonstrated the extent to which an energy device control scheme can be successful without such real-time control. The research went onto demonstrate that not all single phase demand aggregations are candidates for the control methodology and would still require real time monitoring and would therefore require an alternate control technique. Though it has been shown it is possible, based on historical data, to find a set of potential candidate aggregations from the initial complete set of demand aggregations studied. The aggregations that are candidates would have large economical benefits to DNOs and network utility operators. Future work will develop closed loop on-line control methodologies incorporating demand forecasts for the volatile and hard to predict LV network, it will be important to compare these online methodologies, with real time data, to the off-line control scheme presented in this research.

\section{REFERENCES}

[1] G. Strbac, C. Gan, M. Aunedi, and V. Stanojevic, "Benefits of advanced smart metering for demand response based control of distribution networks," Imperial College London, 2010.

[2] M. Wilks, "Demand side response : Conflict between supply and network driven optimisation. A report to DECC," Tech. Rep. November, 2010.

[3] D. Manz, P. Richard, and N. Miller, "Look before you leap," Power and Energy Magazine, IEEE, pp. 75-84, 2012.

[4] H. Jiayi, J. Chuanwen, and X. Rong, "A review on distributed energy resources and MicroGrid," Renewable and Sustainable Energy Reviews, vol. 12, no. 9, pp. 2472-2483, Dec. 2008.

[5] N. Wade, P. Taylor, P. Lang, and P. Jones, "Evaluating the benefits of an electrical energy storage system in a future smart grid," Energy Policy, vol. 38, no. 11, pp. 7180-7188, Nov. 2010.

[6] K. Divya and J. ØStergaard, "Battery energy storage technology for power systems - An overview," Electric Power Systems Research, vol. 79, no. 4, pp. 511-520, Apr. 2009.

[7] A. Mohd and E. Ortjohann, "Challenges in integrating distributed energy storage systems into future smart grid," ISIE 2008, pp. 1627-1632, 2008.

[8] Y. Hida and R. Yokoyama, "Load following operation of NAS battery by setting statistic margins to avoid risks," Power and Energy, pp. 1-5, 2010.

[9] Y. Levron and D. Shmilovitz, "Power systems optimal peak-shaving applying secondary storage," Electric Power Systems Research, vol. 89, pp. 80-84, Aug. 2012.

[10] A. Oudalov, "Sizing and optimal operation of battery energy storage system for peak shaving application," Power Tech, 2007 IEEE, pp. 621-625, 2007

[11] K. M. Chandy, S. H. Low, U. Topcu, and H. Xu, "A simple optimal power flow model with energy storage," 49th IEEE Conference on Decision and Control (CDC), pp. 1051-1057, Dec. 2010.

[12] D. Zhu and G. Hug-Glanzmann, "Real-time control of energy storage devices in future electric power systems," PowerTech, 2011 IEEE Trondheim, pp. 1-7, 2011.

[13] D. Maly and K. Kwan, "Optimal battery energy storage system (BESS) charge scheduling with dynamic programming," Science, Measurement and Technology, vol. 142, no. 6, 1995.

[14] Y. Xu and C. Singh, "Intelligent Control Strategy for Energy Storage in Distribution Systems," Asia-Pacific Power and Energy Engineering Conference, pp. 1-4, Mar. 2012. 
[15] A. Molderink and V. Bakker, "Management and control of domestic smart grid technology," IEEE Transactions On Smart Grid, vol. 1, no. 2, pp. 109-119, 2010.

[16] A. Nourai, V. Kogan, and C. Schafer, "Load leveling reduces T\&D line losses," Power Delivery, IEEE, vol. 23, no. 4, pp. 2168-2173, 2008.

[17] G. Celli, S. Mocci, F. Pilo, and M. Loddo, "Optimal integration of energy storage in distribution networks," 2009 IEEE Bucharest PowerTech, pp. 1-7, Jun. 2009.

[18] I. Koutsopoulos, V. Hatzi, and L. Tassiulas, "Optimal energy storage control policies for the smart power grid," IEEE International Conference on Smart Grid Communications, pp. 475-480, Oct. 2011.

[19] P. Newland, A. Roberts, M. Brandao, and H. Johal, "Energy Storage : Realizing a game changing opportunity," IEEE International Conference on Power System Technology (POWERCON), pp. 1-6, 2012.

[20] D. Westermann and S. Nicolai, "Mid size energy storages in distribution networksoptimal sizing and management," 16th Power Systems Computation Conference, pp. 1-7, 2008.

[21] A. Nourai and C. Schafer, "Changing the electricity game," Power and Energy Magazine, IEEE, pp. 42-47, 2009.

[22] Kema, "Grow-ders," $2005 . \quad$ [Online]. Available: http://growders.eu/index.html

[23] R. Hara, H. Kita, and T. Tanabe, "Testing the technologies," Power and Energy Magazine, IEEE, pp. 77-85, 2009.

[24] Scottish and Southern Energy Power Distribution, "New Thames Valley Vision," 2012. [Online]. Available: http://www.thamesvalleyvision.co.uk/

[25] Irish Social Science Data Archive, "CER Smart Metering Project," 2012. [Online]. Available: http://www.ucd.ie/issda/data/commissionforenergyregulation/

[26] A. Molderink, V. Bakker, and M. Bosman, "A three-step methodology to improve domestic energy efficiency," Innovative Smart Grid Technologies (ISGT), pp. 1-8, 2010.

[27] H. Alfares and M. Nazeeruddin, "Electric load forecasting: literature survey and classification of methods," International Journal of Systems Science, no. 33, pp. 23-34, 2002.

[28] J. Taylor and A. Espasa, "Energy forecasting," International Journal of Forecasting, no. 24, pp. 561-565, 2008.

[29] S. Haben, J. Ward, D. V. Greetham, C. Singleton, and P. Grindrod, "A new error measure for forecasts of household-level, high resolution electrical energy consumption," International Journal of Forecasting, vol. 30 , no. 2, pp. $246-256,2014$.

[30] M. Chaouch, "Clustering-based improvement of nonparametric functional time series forecasting: Application to intra-day household-level load curves," Smart Grid, IEEE Transactions on, vol. 5, no. 1, pp. 411419, 2014.

[31] M. Ghofrani, M. Hassanzadeh, M. Etezadi-Amoli, and M. Fadali, "Clustering-based improvement of nonparametric functional time series forecasting. Application to intraday household-level load curves - In press IEEE Transactions on Smart Grid," IEEE North American Power Symposium (NAPS), 2011.

[32] J. Rawlings, S. Pantula, and D. Dickey, Applied Regression Analysis: A Research Tool, ser. Springer Texts in Statistics. Springer, 2001.

[33] M. Rowe, B. Potter, and W. Holderbaum, "Control Methodologies: Peak Reduction Algorithms For DNO Owned Storage Devices On The Low Voltage Network," IEEE International Smart Grid Technology Conference (ISGT), Europe, 2013.

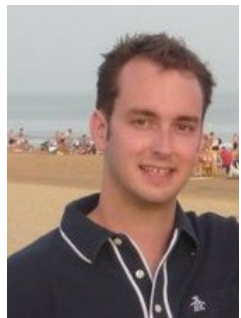

Matthew Rowe (IEEE student member) received his MEng in Electronic Engineering and Cybernetics at the University of Reading in 2011. Currently he is a $\mathrm{PhD}$ student at the University of Reading and working on the LCNF Ofgem funded New Thames Valley Vision Project with Scottish and Southern Energy Power Distribution (SSEPD). Matthews research focuses on the control of storage devices on the Low Voltage Network using demand forecasts.

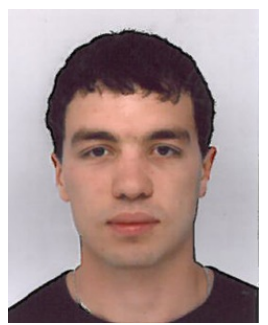

Timur Yunusov (IEEE student member) was born in Kyrgyzstan. He received his MEng in Electronic Engineering and Cybernetics in 2010 from the University of Reading, UK. He is a $\mathrm{PhD}$ student at the School of System Engineering, University of Reading, UK. Current research interests are in decentralised control of distributed generation, energy storage and shiftable demand in located in low voltage distribution network.

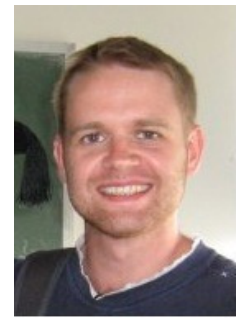

Dr Stephen Haben is a postdoctoral research assistant at the University of Oxford and an academic partner on the New Thames Valley Vision Project as part of Ofgems Low Carbon Network Fund (LCNF). His interests include forecasting, clustering methods, data assimilation, large data analytics, linear algebra and optimisation.

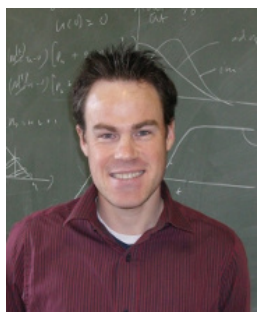

Dr Colin Singleton (IEEE Member) is the Technical Director of CountingLab Limited, a spinout company from the Centre for the Mathematics of Human Behaviour. He has 10 years experience working in Big Data analytics and forecasting. As well as holding a Cambridge $\mathrm{PhD}$ in astrophysics, he has worked for 6 years as a consultant analyst and worked for 3 years as a post doctoral research fellow at the University of Reading, prior to helping set up CountingLab.

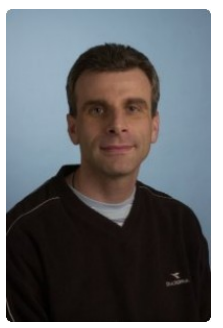

Dr William Holderbaum (IEEE Member) received the Ph.D. degree in automatic control from the University of Lille, Lille, France, in 1999. He was a Research Assistant at the University of Glasgow, Glasgow, U.K., from 1999 to 2001. Currently, he is a Senior Lecturer in the School of Systems Engineering. His research interests are in control theory and its applications. These are mainly focused on geometric control theory in particular Hamiltonian systems and optimisation problems. This research uses the mathematical engineering skills in order to minimise the energy consumption. Further research relates to control theory in the definition of multiple agent systems for distributed network composed of storages, loads and generators.

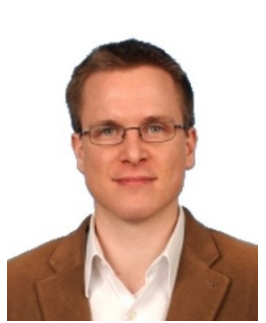

Dr Ben Potter (IEEE Member) completed his MEng degree in Engineering Science at the University of Oxford in 2001 and his $\mathrm{PhD}$ research in the modeling of induction machines at the University of Reading in 2005. Dr Ben Potter subsequently managed research and development activity for several years at Moog Components Group Ltd., including development work on wireless power transfer, before joining the University of Reading as a Researcher and Lecturer in the School of Systems Engineering in 2009. Dr Ben Potters academic research has been focused on energy systems and power electronics, of various flavours, for over twelve years, with applications including energy storage, electric machines, wind turbines and wireless power transfer. In 2010, Dr. Ben Potter founded the Energy Research Lab within the School of Systems Engineering, and this lab focuses on the development of control methods for the new generation of energy networks the smart grid to ensure that the integration of active elements such as renewable energy resources, electric vehicles and energy storage devices will have positive impacts for both network operators and end users. 